October 2016

\title{
Radiotherapy for localised Ewing's sarcoma in children
}

masood umer

Aga Khan University, masood.umer@aku.edu

Irfan Qadir

Aga Khan University, irfan.qadir@aku.edu

Ahmed Nadeem Abbasi

Aga Khan University, nadeem.abbasi@aku.edu

Zohra Lassi

Aga Khan University

Follow this and additional works at: http://ecommons.aku.edu/pakistan_fhs_mc_surg_surg Part of the Orthopedics Commons

\section{Recommended Citation}

umer, m., Qadir, I., Abbasi, A. N., Lassi, Z. (2016). Radiotherapy for localised Ewing's sarcoma in children. The Cochrane Library, 1-12. Available at: http://ecommons.aku.edu/pakistan_fhs_mc_surg_surg/247 


\section{Radiotherapy for localised Ewing's sarcoma in children: Protocols}

Chapter in Cohrane Database of Systematic Reviews · October 2016

DOI: 10.1002/14651858.CD011105.pub2

CITATIONS

0

5 authors, including:

\section{Masood Umer}

Aga Khan University Hospital, Karachi

72 PUBLICATIONS 482 CITATIONS

SEE PROFILE

\section{Nadeem Abbasi}

Aga Khan University, Pakistan

42 PUBLICATIONS 89 CITATIONS

SEE PROFILE
READS

16

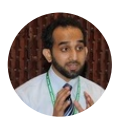

\section{Irfan Qadir}

Aga Khan University Hospital, Karachi

25 PUBLICATIONS 146 CITATIONS

SEE PROFILE

\section{Zohra S Lassi}

University of Adelaide

106 PUBLICATIONS 1,218 CITATIONS

SEE PROFILE

Some of the authors of this publication are also working on these related projects:

Project Identification of central and peripheral pain mechanisms in Osteoarthritis View project 


\section{Radiotherapy for localised Ewing's sarcoma in children (Protocol)}

Umer M, Qadir I, Abbasi N, Das JK, Lassi ZS

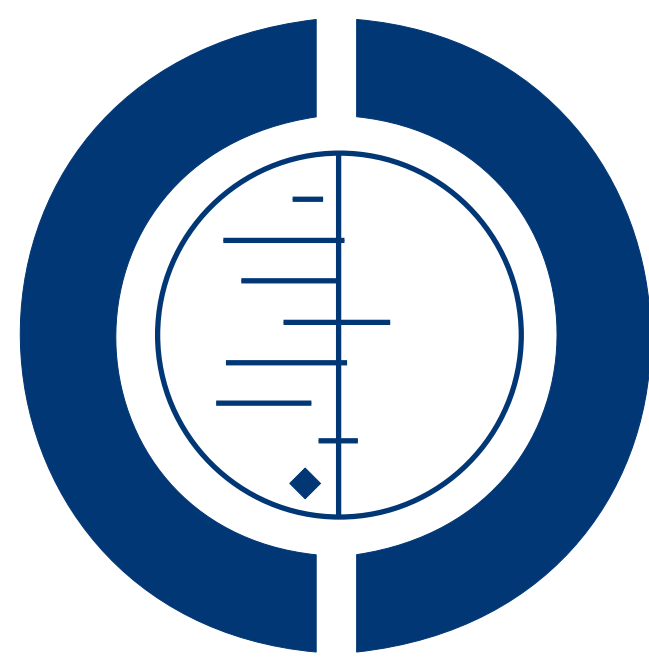

THE COCHRANE COLLABORATION $^{\circledR}$

This is a reprint of a Cochrane protocol, prepared and maintained by The Cochrane Collaboration and published in The Cochrane Library 2014, Issue 5

http://www.thecochranelibrary.com

\section{WILEY}


TABLE OF CONTENTS

HEADER . . . . . . . . . . . . . . . . . . . . . . . . . . . . . . . . . . . . . 1

ABSTRACT . . . . . . . . . . . . . . . . . . . . . . . . . . . . . . . . . . . . . . . . . . . . . . . .

BACKGROUND . . . . . . . . . . . . . . . . . . . . . . . . . . . . . . . . . . . .

OBJECTIVES . . . . . . . . . . . . . . . . . . . . . . . . . . . . . . . . . . . . . . . . . .

METHODS . . . . . . . . . . . . . . . . . . . . . . . . . . . . . . . . . . . . . .

ACKNOWLEDGEMENTS . . . . . . . . . . . . . . . . . . . . . . . . . . . . . . . . . . . . .

REFERENCES . . . . . . . . . . . . . . . . . . . . . . . . . . . . . . . . . . . . . . . 5

APPENDICES . . . . . . . . . . . . . . . . . . . . . . . . . . . . . . . . . . . . . . 7

DECLARATIONS OF INTEREST . . . . . . . . . . . . . . . . . . . . . . . . . . . . . . . . . . . . . 9 


\title{
[Intervention Protocol]
}

\section{Radiotherapy for localised Ewing's sarcoma in children}

\author{
Masood Umer ${ }^{1}$, Irfan Qadir ${ }^{1}$, Nadeem Abbasi ${ }^{2}$, Jai K Das ${ }^{3}$, Zohra S Lassi ${ }^{3}$ \\ ${ }^{1}$ Department of Surgery, Aga Khan University Hospital, Karachi, Pakistan. ${ }^{2}$ Department of Radiation Oncology, Aga Khan University \\ Hospital, Karachi, Pakistan. ${ }^{3}$ Division of Women and Child Health, Aga Khan University Hospital, Karachi, Pakistan \\ Contact address: Masood Umer, Department of Surgery, Aga Khan University Hospital, Stadium Road, Karachi, 74800, Pakistan. \\ masood.umer@aku.edu.
}

Editorial group: Cochrane Childhood Cancer Group.

Publication status and date: New, published in Issue 5, 2014.

Citation: Umer M, Qadir I, Abbasi N, Das JK, Lassi ZS. Radiotherapy for localised Ewing's sarcoma in children. Cochrane Database of Systematic Reviews 2014, Issue 5. Art. No.: CD011105. DOI: 10.1002/14651858.CD011105.

Copyright (C) 2014 The Cochrane Collaboration. Published by John Wiley \& Sons, Ltd.

\begin{abstract}
A B S T R A C T
This is the protocol for a review and there is no abstract. The objectives are as follows:

(a) To determine the effectiveness of radiotherapy in localised Ewing's sarcoma in children for event-free survival and overall survival when compared to children undergoing surgery only.
\end{abstract}

(b) To determine the role of radiotherapy for Ewing's sarcoma on risk of growth arrest and second malignancies

\section{B A C K G RO U N D}

\section{Description of the condition}

Ewing's sarcoma family tumours (EWS), which include classic Ewing's sarcoma in addition to primitive neuroectodermal tumour and Askin tumour, are the second most common variety of primary bone cancer in adolescents and young adults (Subbiah 2009). Annual incidence of Ewing's sarcoma is low ( 0.6 per million) before age five but rises in concordance with the arrival of puberty to approach a peak rate of 5 per million, accounting for 3\% of paediatric cancers. Most common sites of tumour are pelvis, femur, tibia and chest wall (Ludwig 2008).

The single most important criterion considered when planning treatment of people with Ewing's sarcoma is the presence or absence of metastatic disease (Ludwig 2008). The treatment algorithm for localised disease consists generically of three stages: initial cytoreduction to eradicate micrometastatic disease and facili- tate effective local control measures with wide negative margins; definitive radiation or surgical therapy to eradicate all known disease; and consolidation with adjuvant chemotherapy to reduce the likelihood of tumour recurrence. This multi-disciplinary care incorporating advances in diagnosis, surgery, chemotherapy, and radiation has substantially improved the survival rate of people with localised Ewing's sarcoma to nearly 70\% (Subbiah 2009).

\section{Description of the intervention}

Known to be radiation-sensitive since the earliest clinical reports, localised Ewing's sarcoma had been treated throughout most of the 20th century primarily with radiation (Indelicato 2008a; Indelicato 2008b; Laskar 2008). Today, this modality is used less frequently as a definitive measure, given the potential morbidities of this approach (secondary malignancies and adverse effects on bone growth) in children (Ludwig 2008). Contemporary surgical advances, that preserve limb function even after excision with a 
wide margin, provide a higher survival rate and reduce the need for adjuvant radiation (Ludwig 2008). However, there is ongoing debate as to whether surgery and radiotherapy are comparable for local control. Though there are no randomised controlled studies that directly compare surgery to radiation for definitive local control, at least four studies report local failure rates nearly three-fold higher in participants treated with radiation alone (30\% failure) compared to surgery alone (10\% or less) (Bacci 2004; Donaldson 1998; Krasin 2004; Schuck 2003). These results should be interpreted with caution as they are retrospective studies susceptible to inherent bias in the selection of one therapy over another. The choice of local therapy is influenced by multiple clinical characteristics such as age, tumour location, size, surgical margins, response to chemotherapy, availability of surgical or radiotherapy resources, anticipated morbidity and patient preference. People referred for surgery have tumours which are usually small, peripheral in location, and follow a good response to induction chemotherapy. Tumours requiring radiotherapy are often large, central in location, and follow a poor response to induction chemotherapy (Ludwig 2008).

Several radiotherapy parameters have been studied, including treatment volume, timing, and dose. For dose, there have been conflicting results regarding its impact on local control. St Jude Children's Research Hospital (SJCRH) reported local control of $58 \%$ following 30 to 60 Gy (Arai 1991). The SJCRH investigators used lower doses of 30 to $36 \mathrm{~Gy}$, for those who responded to chemotherapy, but 50 to 60 Gy for those with no response or progressive disease during chemotherapy. Improved outcome among chemotherapy responders was recorded. They also showed local tumour control is related to the size of the primary tumour. Those with a tumour of less than $8 \mathrm{~cm}$ in diameter who received a median radiation dose of 35 Gy had $90 \%$ local control, while for those with a tumour of $8 \mathrm{~cm}$ or more, local control dropped to $52 \%$ (Arai 1991). On the other hand, the Intergroup Ewing Sarcoma Study (IESS) showed no dose response between 30 and $65 \mathrm{~Gy}$; local control was achieved in 6/6 (100\%) with 30 to 39.9 Gy, 37/43 (86\%) with 40 to $49.9 \mathrm{~Gy}, 80 / 91$ (88\%) with 50 to 59.9 Gy and 50/53 (94\%) with 60 Gy or more (Razek 1980). Similarly, a recent paper from Memorial Sloan Kettering Cancer Center did not show a radiotherapy dose response (La 2006).

There are concerns about potential late effects and second malignancies in long-term survivors. People treated for Ewing's sarcoma have a significantly higher risk of developing subsequent neoplasms than the general population. Treatment-related acute myeloid leukaemia (AML) and myelodysplastic syndrome (MDS) have generally been reported to occur in $1 \%$ to $2 \%$ of survivors of Ewing's sarcoma (Fuchs 2003; Goldsby 2008), although some dose-intensive regimens appear to be associated with a higher risk of haematological malignancy (Bhatia 2007; Kushner 1998; Navid 2008). Treatment-related AML and MDS arise most commonly at two to five years following diagnosis.

Survivors of Ewing's sarcoma remain at increased risk of develop- ing a subsequent solid tumour throughout their lifetime. Sarcomas usually occur within the prior radiation field (Hawkins 1996; Kuttesch 1996). The risk of developing a sarcoma following radiation therapy is dose-dependent, with higher doses associated with an increased risk of sarcoma development (Fuchs 2003; Goldsby 2008). The cumulative incidence of subsequent neoplasms in children treated for Ewing's sarcoma between 1970 and 1986 at 25 years after diagnosis was $9.0 \%$ (95\% confidence interval 5.8 to 12.2). Most of these people received radiation therapy; comparable long-term data do not yet exist for significant numbers of people who did not receive radiation therapy (Ginsberg 2010).

\section{Why it is important to do this review}

Current opinion favouring surgery is based on retrospective analysis and should therefore be interpreted with caution. This review will provide a systematic analysis of the available evidence for the effectiveness of radiotherapy for local control in localised Ewing's sarcoma compared to surgery alone, and should therefore contribute to facilitating evidence-based treatment decisions in a complex field.

\section{O B J E C T I V E S}

(a) To determine the effectiveness of radiotherapy in localised Ewing's sarcoma in children for event-free survival and overall survival when compared to children undergoing surgery only.

(b) To determine the role of radiotherapy for Ewing's sarcoma on risk of growth arrest and second malignancies

\section{METHODS}

\section{Criteria for considering studies for this review}

\section{Types of studies}

Randomised controlled trials and quasi-randomised controlled trials comparing radiotherapy and surgery as part of treatment for localised Ewing's sarcoma in children.

\section{Types of participants}

The review will include trials on children (less than 18 years of age) with a diagnosis of localised Ewing's sarcoma established by histopathology and a negative metastatic workup. There will be no restriction by participant gender or ethnic group. We will also consider studies which include participants younger than 18 years 
and older than 18 years. For such studies we will assess if separate data are available for participants younger than 18 years. If data are not given separately for our review population we will not include these studies in the review.

\section{Types of interventions}

We will include trials which compare radiotherapy versus surgery for localised Ewing's sarcoma in addition to neo-adjuvant and adjuvant chemotherapy. The type and dose of chemotherapy must be the same in both intervention and control groups.

\section{Types of outcome measures}

\section{Primary outcomes}

Event-free survival (EFS) is defined as the time from the date of diagnosis until the event or last participant contact, at which time the participant is censored. An 'event' will be defined as stated by the authors of included studies. Overall survival is defined as the time from the date of diagnosis until the death of the participant from any cause.

\section{Secondary outcomes}

Risk of second malignancy, defined as cancer that develops after treatment for a first cancer. The risk of growth retardation will be determined in terms of limb length discrepancy.

We will investigate the above-mentioned outcomes, although these outcome measures are not used as criteria for inclusion of studies. Studies will be chosen purely on their relevance to the study objective.

\section{Search methods for identification of studies}

We will not impose any language or date of publication restriction. We will update searches every two years.

\section{Electronic searches}

We will search the following electronic databases for primary studies: the Cochrane Central Register of Controlled Trials (CENTRAL, The Cochrane Library, latest issue), MEDLINE/PubMed (from 1945 to present), EMBASE/Ovid (from 1980 to present). The Cochrane Childhood Cancer Group (CCG) will run the searches in these databases.

The search strategies for the different electronic databases (using a combination of controlled vocabulary and text words) are shown in the appendices (Appendix 1; Appendix 2; Appendix 3).

\section{Searching other resources}

We will locate information about trials not identified in CENTRAL, MEDLINE and EMBASE, either published or unpublished, by searching the reference lists of relevant trial articles and review articles.

We also plan to scan the conference proceedings of the International Society of Paediatric Oncology (SIOP) (from 2009 to present), the European Musculo-Skeletal Oncology Society (E.M.S.O.S.) from 2012 onwards, the International Society of Limb Salvage (ISOLS) (2011 and 2013) and the American Society of Clinical Oncology (ASCO; meetinglibrary.asco.org/abstracts) (from 2009 to present).

We will search for ongoing trials by scanning the following trial registers:

www.clinicaltrials.gov

www.who.int/ictrp/en/

We will also handsearch the journals in which the included studies most frequently appear. We will search the top five journals (according to the number of included studies provided) for the last 12 months.

We will contact authors of relevant papers regarding any further published or unpublished work.

\section{Data collection and analysis}

\section{Selection of studies}

Two review authors (IQ and JD) will download all titles and abstracts retrieved by electronic searching to the reference management database Endnote, will remove duplicates and examine the remaining references independently. These two review authors will screen titles and abstracts of references identified from the search and will eliminate articles that are obviously not relevant to the search question. We will assess all other references in full text. Each review author will then independently determine if these trials are eligible for inclusion, resolving disagreements about inclusion by discussion and if necessary by recourse to a third review author (MU). We will contact study authors for further details when papers contain insufficient information to make a decision about eligibility. We will document reasons for exclusion. We will include a flow chart/diagram of the search in the review.

\section{Data extraction and management}

Two review authors (IQ and NA, not masked to the study details) will independently perform extraction of data from the included trials, using a data extraction form designed and pilot-tested. We will extract each trial component, such as study design, participants, setting, intervention and control, co-interventions, outcomes and results, follow-up duration, funding source details, the declaration of interests for the primary investigators, and risk of 
bias domains, and will present them in a table of Characteristics of Included Studies.

The review authors will compare results and resolve disagreements by discussion until a consensus is reached. If there is no consensus, a third review author (MU) will settle the discrepancies. We will document relevant disagreements. We will write to the study authors for any missing, incomplete or unclear data. One review author (IQ) will enter the data in to Review Manager 5 software (RevMan 2012) and another (JD) will check the data entered manually. A third author (ZL) will check the whole process.

\section{Dealing with duplicate publications}

If there are multiple publications for one study, we will use the one with the most recent data. We may use earlier publications for extracting information on baseline characteristics or methodology.

\section{Assessment of risk of bias in included studies}

Two review authors (IQ and JD) will independently assess the risk of bias for each study (that is, selection bias, performance bias, detection bias (for each outcome separately), attrition bias (for each outcome separately), reporting bias and other potential bias) using the 'Risk of bias' items as described in the module of the Childhood Cancer Group (Module CCG), which are based on the Cochrane Handbook for Systematic Reviews of Interventions (Higgins 2011). We will contact study authors if relevant data are missing. We will take the risk of bias in included studies into account in the interpretation of the review's results.

\section{Measures of treatment effect}

Measures of treatment effect will depend on the types of data presented in the individual studies.

1) Hazard ratios (HRs) for time-to-event variables (event-free survival and overall survival). We will use Parmar's method if hazard ratios have not been explicitly presented in the study (Parmar 1998).

2) Risk ratios (RRs) for dichotomous variables (risk of second malignancy and growth arrest).

\section{Unit of analysis issues}

\section{Studies with more than two treatment groups}

If we identify studies with more than two intervention groups (multi-arm studies), we will where possible combine groups to create a single pair-wise comparison or use the methods set out in the Cochrane Handbook for Systematic Reviews of Interventions (Higgins 2011) to avoid double-counting study participants. For the subgroup analyses, when the control group is shared by two or more study arms, we will divide the control group (events and total population) over the number of relevant subgroups to avoid double-counting the participants.

\section{Dealing with missing data}

As stated before, when relevant data regarding study selection, data extraction and risk of bias assessment are missing, we will attempt to contact the study authors to retrieve the missing data. If after 30 days we cannot obtain this information, we will use the available information and perform an 'available case analysis' instead of an intention-to-treat analysis if possible. An 'available case analysis' includes data on only those participants whose results are known, using as a denominator the total number of people who had data recorded for the particular outcome in question (the participants are analysed according to the group to which they were randomised). If we do not have enough information to include a certain outcome in the analysis, we will declare this.

We will describe missing outcomes of the included studies by reporting proportions of randomised participants for whom no outcome data were obtained (with reasons) by outcome and by randomised group. We will address the potential impact of the missing outcomes on the results of the included studies in the assessment of risk of bias and we will describe in the Discussion section its impact on the findings of the review. We will perform sensitivity analyses to assess how sensitive results are to changes in the assumptions made in the 'available case analysis.'

\section{Assessment of heterogeneity}

We will investigate meta-analysis results for clinical and statistical heterogeneity.

We will check for heterogeneity by considering the following factors:

1. The clinical or methodological characteristics of the studies.

2. The results of the $\mathrm{I}^{2}$ statistic for the quantification of the heterogeneity. The $\mathrm{I}^{2}$ statistic describes the percentage of total variation across studies that is due to heterogeneity rather than to chance (Higgins 2003). We will judge the importance of the observed value of $\mathrm{I}^{2}$ depending on the magnitude and direction of effects and the strength of evidence for heterogeneity (moderate to high heterogeneity will be defined as $\mathrm{I}^{2}$ of $50 \%$ or more).

If we find substantial heterogeneity, we will not pool the data, but will attempt to explain the observed differences by examining individual study characteristics and by performing subgroup analyses.

\section{Assessment of reporting biases}

In addition to the evaluation of reporting bias as described in the 'Assessment of risk of bias' section, we will investigate reporting biases (such as publication bias) using funnel plots, provided there are 10 or more studies in the meta-analysis. We will assess funnel plot asymmetry visually, and use formal tests for funnel plot asymmetry. For continuous outcomes we will use the test proposed by Egger 1997, and for dichotomous outcomes we will use the test proposed by Harbord 2006. If we detect asymmetry in any 
of these tests or if a visual assessment suggests it, we will perform exploratory analyses to investigate it.

\section{Data synthesis}

We will carry out statistical analysis using the Review Manager 5 software (RevMan 2012). We will use a fixed-effect meta analysis for combining data where it is reasonable to assume that studies are estimating the same underlying treatment effect, i.e. where trials are examining the same intervention, and we judge the outcome definitions, study population and methods to be sufficiently similar between studies. If there is clinical heterogeneity (regarding interventions, study populations, methods and outcome definitions) we will not pool the results. We will descriptively summarise studies for which pooling of results is not possible. We will use the fixed-effect model if $\mathrm{I}^{2}$ is $50 \%$ or less. If $\mathrm{I}^{2}$ is greater than $50 \%$ we will use a random-effects model. We will present the results as the average treatment effect with $95 \%$ confidence intervals, and the estimate of $\mathrm{I}^{2}$. We will set out the main findings of the review in a 'Summary of findings' table prepared using the GRADE approach using GRADE profiler software (Guyatt 2008). We will list all outcomes for each comparison with estimates of relative effects along with the number of participants and studies contributing data for those outcomes. For each individual outcome, we will assess the quality of the evidence using the GRADE approach, which involves consideration of within-study risk of bias (methodological quality), directness of evidence, heterogeneity, precision of effect estimates and risk of publication bias. We will rate the quality of the body of evidence for each key outcomes as 'high', 'moderate', 'low' or 'very low'.

\section{Subgroup analysis and investigation of heterogeneity}

We will undertake subgroup analysis, grouping the trials by radiation dose ( $<60$ Gy versus $\geq 60 \mathrm{~Gy}$ ). The usual radiation dose in Ewing's sarcoma in 40 to $60 \mathrm{~Gy}$. Higher radiation doses are associated with increased risk of adverse events, including second malignancies, and lower doses are associated with suboptimal response (Kuttesch 1996).

We will assess subgroup differences by interaction tests available within RevMan. We will report the results of subgroup analyses quoting the $\mathrm{Chi}^{2}$ statistic and $\mathrm{P}$ value, and the interaction test $\mathrm{I}^{2}$ value.

\section{Sensitivity analysis}

Besides the sensitivity analyses mentioned earlier in this protocol, we will conduct a repeat of the primary meta-analysis, excluding studies at high risk or uncertain risk of bias. In this sensitivity analysis, we will only include studies that have a low risk of bias in all key domains for the estimates of treatment effect.

\section{ACKNOW LEDGEMENTS}

We thank Edith Leclercq for her help with developing the search strategies for the CENTRAL, MEDLINE and EMBASE databases. The editorial base of the Cochrane Childhood Cancer Group is funded by Stichting Kinderen Kankervrij (KiKa). We also thank Dr. H. van den Berg (Department of Pediatric Oncology, Emma Children's Hospital/Academic Medical Center, Amsterdam, the Netherlands) and two undisclosed persons who kindly agreed to peer review our protocol.

\section{REFEREN CES}

\section{Additional references}

\section{Arai 1991}

Arai Y, Kun LE, Brooks MT, Fairclough DL, Fontanesi J, Meyer WH, et al.Ewing's sarcoma: local tumor control and patterns of failure following limited-volume radiation therapy. International Journal of Radiation Oncology, Biology, Physics 1991;21(6):1501-8.

Bacci 2004

Bacci G, Forni C, Longhi A, Ferrari S, Donati D, De Paolis M, et al.Long-term outcome for patients with nonmetastatic Ewing's sarcoma treated with adjuvant and neoadjuvant chemotherapies. 402 patients treated at Rizzoli between 1972 and 1992. European Journal of Cancer 2004; 40(1):73-83.

Bhatia 2007

Bhatia S, Krailo MD, Chen Z, Burden L, Askin FB, Dickman PS, et al.Therapy-related myelodysplasia and acute myeloid leukemia after Ewing sarcoma and primitive neuroectodermal tumor of bone: A report from the Children's Oncology Group. Blood 2007;109(1):46-51.

Donaldson 1998

Donaldson SS, Torrey M, Link MP, Glicksman A, Gilula L, Laurie F, et al.A multidisciplinary study investigating radiotherapy in Ewing's sarcoma: end results of POG \# 8346. Pediatric Oncology Group. International Journal of Radiation Oncology, Biology, Physics 1998;42(1):125-35.

Egger 1997

Egger M, Davey Smith G, Schneider M, Minder C. Bias in meta-analysis detected by a simple, graphical test. $B M J$ (Clinical research ed.) 1997;315(7109):629-34.

Fuchs 2003

Fuchs B, Valenzuela RG, Petersen IA, Arndt CA, Sim FH. Ewing's sarcoma and the development of secondary 
malignancies. Clinical Orthopaedics and Related Research 2003;Oct(415):82-9.

\section{Ginsberg 2010}

Ginsberg JP, Goodman P, Leisenring W, Ness KK, Meyers PA, Wolden SL, et al.Long-term survivors of childhood Ewing sarcoma: report from the childhood cancer survivor study. Journal of the National Cancer Institute 2010;102 (16): 1272-83

\section{Goldsby 2008}

Goldsby R, Burke C, Nagarajan R, Zhou T, Chen Z, Marina $\mathrm{N}$, et al.Second solid malignancies among children, adolescents, and young adults diagnosed with malignant bone tumors after 1976: follow-up of a Children's Oncology Group cohort. Cancer 2008;113(9):2597-604.

Guyatt 2008

Guyatt GH, Oxman AD, Vist GE, Kunz R, Falck-Ytter Y, Alonso-Coello P, et al.GRADE: an emerging consensus on rating quality of evidence and strength of recommendations. BMJ (Clinical research ed.) 2008;336(7650):924-6.

\section{Harbord 2006}

Harbord RM, Egger M, Sterne JA. A modified test for small-study effects in meta-analyses of controlled trials with binary endpoints. Statistics in Medicine 2006;25(20): 3443-57.

\section{Hawkins 1996}

Hawkins MM, Wilson LM, Burton HS, Potok MH, Winter DL, Marsden HB, et al.Radiotherapy, alkylating agents, and risk of bone cancer after childhood cancer. Journal of the National Cancer Institute 1996;88(5):270-8.

\section{Higgins 2003}

Higgins JPT, Thompson SG, Deeks JJ, Altman DG. Measuring inconsistency in meta-analyses. BMJ 2003;327 (7414):557-60.

\section{Higgins 2011}

Higgins JPT, Green S (editors). Cochrane Handbook for Systematic Reviews of Interventions Version 5.1.0 [updated March 2011]. The Cochrane Collaboration, 2011. Available from www.cochrane-handbook.org. ..

Indelicato 2008a

Indelicato DJ, Keole SR, Shahlaee AH, Shi W, Morris CG, Marcus RB Jr. Definitive radiotherapy for ewing tumors of extremities and pelvis: long-term disease control, limb function, and treatment toxicity. International Journal of Radiation Oncology, Biology, Physics 2008;72(3):871-7.

\section{Indelicato 2008b}

Indelicato DJ, Keole SR, Shahlaee AH, Shi W, Morris CG, Gibbs CP Jr, et al.Long-term clinical and functional outcomes after treatment for localized Ewing's tumor of the lower extremity. International Journal of Radiation Oncology, Biology, Physics 2008;70(2):501-9.

\section{Krasin 2004}

Krasin MJ, Rodriguez-Galindo C, Billups CA, Davidoff AM, Neel MD, Merchant TE, et al.Definitive irradiation in multidisciplinary management of localized Ewing sarcoma family of tumors in pediatric patients: outcome and prognostic factors. International Journal of Radiation

Oncology, Biology, Physics 2004;60(3):830-8.

\section{Kushner 1998}

Kushner BH, Heller G, Cheung NK, Wollner N, Kramer $\mathrm{K}$, Bajorin $\mathrm{D}$, et al.High risk of leukemia after short-term dose-intensive chemotherapy in young patients with solid tumors. Journal of Clinical Oncology 1998;16(9):3016-20.

\section{Kuttesch 1996}

Kuttesch JF Jr, Wexler LH, Marcus RB, Fairclough D, Weaver-McClure $\mathrm{L}$, White $\mathrm{M}$, et al.Second malignancies after Ewing's sarcoma: radiation dose-dependency of secondary sarcomas. Journal of Clinical Oncology 1996;14 (10):2818-25.

\section{La 2006}

La TH, Meyers PA, Wexler LH, Alektiar KM, Healey JH, Laquaglia MP, et al.Radiation therapy for Ewing's sarcoma: results from Memorial Sloan-Kettering in the modern era. International Journal of Radiation Oncology, Biology, Physics 2006;64(2):544-50.

Laskar 2008

Laskar S, Mallick I, Gupta T, Muckaden MA. Post-operative radiotherapy for Ewing sarcoma: when, how and how much?. Pediatric Blood and Cancer 2008;51(5):575-80.

Ludwig 2008

Ludwig JA. Ewing sarcoma: historical perspectives, current state-of-the-art, and opportunities for targeted therapy in the future. Current Opinion in Oncology 2008;20(4):412-8.

\section{Module CCG}

Kremer LCM, van Dalen EC, Moher D, Caron HN. Cochrane Childhood Cancer Group. The Cochrane Library 2013 , issue 8 .

\section{Navid 2008}

Navid F, Billups C, Liu T, Krasin MJ, Rodriguez-Galindo C. Second cancers in patients with the Ewing sarcoma family of tumours. European Journal of Cancer 2008;44(7):983-91.

\section{Parmar 1998}

Parmar MK, Torri V, Stewart L. Extracting summary statistics to perform meta-analyses of the published literature for survival endpoints. Statistics in Medicine 1998;17(24): 2815-34.

\section{Razek 1980}

Razek A, Perez CA, Tefft M, Nesbit M, Vietti T, Burgert EO $\mathrm{Jr}$, et al.Intergroup Ewing's Sarcoma Study: local control related to radiation dose, volume, and site of primary lesion in Ewing's sarcoma. Cancer 1980;46(3):516-21.

RevMan 2012

The Nordic Cochrane Centre, The Cochrane Collaboration Review Manager (RevMan). 5.2. Copenhagen: The Nordic Cochrane Centre, The Cochrane Collaboration, 2012.

\section{Schuck 2003}

Schuck A, Ahrens S, Paulussen M, Kuhlen M, Konemann $S$, Rube C, et al.Local therapy in localized Ewing tumors: results of 1058 patients treated in the CESS 81, CESS 86, and EICESS 92 trials. International Journal of Radiation Oncology, Biology, Physics 2003;55(1):168-77. 


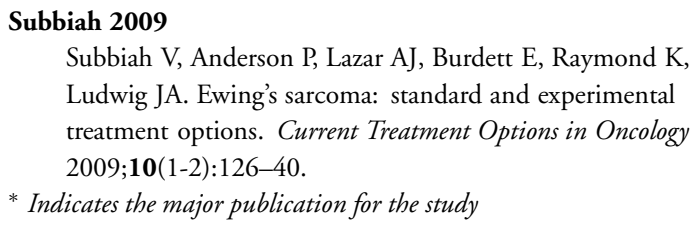

\section{A P P E N D I C E S}

\section{Appendix I. Search strategy for Cochrane Central Register of Controlled Trials (CENTRAL)}

1. ForEwing's sarcoma the following text words will be used: ewing OR ewings OR ewing* OR ewing sarcoma OR ewing's sarcoma OR primitive neuroepithelial tumor OR primitive neuroepithelial tumors OR primitive neuroectodermal tumor OR primitive neuroectodermal tumors OR primitive neuroepithelial neoplasm OR primitive neuroepithelial neoplasms OR primitive neuroectodermal neoplasms OR primitive neuroectodermal neoplasm OR PNET OR PNETs OR sarcoma, soft tissue OR sarcomas, soft tissue OR soft tissue sarcoma OR soft tissue sarcomas OR "askin tumor" OR "askin tumors" OR "askin tumour" OR "askin tumours" OR “askin tumor*” OR "askin tumour*”

2. For Radiotherapy the following text words will be used:

Radiotherapy OR radiation OR radiation therapy OR irradiation OR irradiat* OR radiation injuries OR injuries, radiation OR injury, radiation $\mathrm{OR}$ radiation injury $\mathrm{OR}$ radiation syndrome $\mathrm{OR}$ radiation syndromes $\mathrm{OR}$ syndrome radiation $\mathrm{OR}$ radiation sickness $\mathrm{OR}$ radiation sicknesses OR sickness radiation OR radiation* $\mathrm{OR}$ irradiation OR radiations

3. For Surgery the following text words will be used:

surgery OR surger* OR surgeri* OR surgical OR surgical* OR operation OR operat* OR operate OR operative procedure

Final search

1 AND (2 OR 3)

$\left[{ }^{*}=\right.$ zero or more characters $]$

The search will be performed in title, abstract or keywords

\section{Appendix 2. Search strategy for PubMed}

1. For Ewing's sarcoma the following MeSH headings and text words will be used: ewing[tiab] OR ewings[tiab] OR ewing*[tiab] OR ewing sarcoma[tiab] OR ewing's sarcoma OR "primitive neuroepithelial tumor" OR "primitive neuroepithelial tumors" OR "primitive neuroectodermal tumor" OR "primitive neuroectodermal tumors" OR "primitive neuroepithelial neoplasm” OR "primitive neuroepithelial neoplasms" OR "primitive neuroectodermal neoplasms" OR "primitive neuroectodermal neoplasm" OR "PNET” OR "PNETs" OR (“askin tumor" OR “askin tumors” OR "askin tumour” OR "askin tumours” OR “askin tumor*” OR "askin tumour*”)

2. ForRadiotherapy the following MeSH headings and text words will be used:

Radiotherapy OR radiation OR radiation* OR radiations OR radiation therapy OR irradiation OR irradiat* OR radiation injuries OR injuries, radiation $\mathrm{OR}$ injury, radiation $\mathrm{OR}$ radiation injury $\mathrm{OR}$ radiation syndrome $\mathrm{OR}$ radiation syndromes $\mathrm{OR}$ syndrome radiation OR radiation sickness $\mathrm{OR}$ radiation sicknesses $\mathrm{OR}$ sickness radiation

3. For Surgery the following MeSH headings and text words will be used: surgery OR surger* OR surgeri* OR surgical OR surgical* OR Surgical Procedures, Operative[MeSH] OR operation OR operat* OR operate OR operative procedure

4. For RCTs and CCTs the following MeSH headings and text words will be used:

((randomized controlled trial[pt]) OR (controlled clinical trial[pt]) OR (randomized[tiab]) OR (placebo[tiab]) OR (drug therapy[sh]) OR (randomly[tiab]) OR (trial[tiab]) OR (groups[tiab])) AND (humans[mh])

Final search

1 AND (2 OR 3) AND 4

Radiotherapy for localised Ewing's sarcoma in children (Protocol)

Copyright @ 2014 The Cochrane Collaboration. Published by John Wiley \& Sons, Ltd. 
$\left[\mathrm{pt}=\right.$ publication type; tiab = title, abstract; $\mathrm{sh}=$ subject heading; $\mathrm{mh}=\mathrm{MeSH}$ term; ${ }^{*}=$ zero or more characters; $\mathrm{RCT}=$ randomized controlled trial; CCT $=$ controlled clinical trial]

\section{Appendix 3. Search strategy for EMBASE (OVID)}

1. For Ewing's sarcoma the following Emtree terms and text words will be used:

1. (ewing or ewings or ewing\$).ti,ab.

2. exp Ewing sarcoma/

3. (ewing sarcoma or ewing's sarcoma).mp.

4. (PNET or PNETs).mp. [mp=title, abstract, subject headings, heading word, drug trade name, original title, device manufacturer, drug manufacturer, device trade name, keyword]

5. (peripheral neuroectodermal tumor or peripheral neuroectodermal tumors).mp.

6. (primitive neuroectodermal tumor or primitive neuroectodermal tumors).mp.

7. (Primitive Neuroepithelial Tumor or Primitive Neuroepithelial Tumors).mp.

8. neuroectoderm tumor/ or neuroepithelioma/

9. (Primitive Neuroepithelial Neoplasm or Primitive Neuroepithelial Neoplasms).mp.

10. exp soft tissue sarcoma/

11. (soft tissue sarcoma or soft tissue sarcomas).mp.

12. (askin tumor or askin tumors or askin tumour or askin tumours or askin tumor\$ or askin tumor\$).mp.

13. or/ $1-12$

2. For Radiotherapy the following Emtree terms and text words will be used:

1. Radiotherapy.mp. or exp radiotherapy/

2. exp radiation/ or radiation.mp.

3. (radiation* or radiations).mp.

4. radiation therapy.mp.

5. radiation injuries.mp. or exp radiation injury/

6. radiation injury.mp.

7. exp irradiation/

8. (irradiation or irradiat\$).mp.

9. exp radiation sickness/

10. (radiation syndrome or radiation syndromes).mp.

11. (syndrome radiation or radiation sickness or radiation sicknesses or sickness radiation).mp.

12. or/1-11

3. For Surgery the following Emtree terms and text words will be used:

1. exp surgery/

2. (surgery or surger\$ or surgeri\$).mp.

3. (surgical or surgical\$).mp.

4. surgical technique/

5. (surgical procedure or surgical procedures).mp.

6. (operate or operat $\$$ or operative procedure).mp.

7. operation/

8. or/1-6

4. For RCTs and CCTs the following Emtree terms and text words will be used:

1. Randomized Controlled Trial/

2. Controlled Clinical Trial/

3. randomized.ti,ab.

4. placebo.ti,ab.

5. randomly.ti,ab.

6. trial.ti,ab.

7. groups.ti,ab.

8. drug therapy.sh.

9. or/1-8

Radiotherapy for localised Ewing's sarcoma in children (Protocol)

Copyright (C) 2014 The Cochrane Collaboration. Published by John Wiley \& Sons, Ltd. 
10. Human/

11.9 and 10

Final search

1 AND (2 OR 3) AND 4

[mp=title, abstract, subject headings, heading word, drug trade name, original title, device manufacturer, drug manufacturer, device trade name, keyword; sh = subject heading; ti,ab = title, abstract; / = Emtree term; $\$=$ zero or more characters ; RCT = randomized controlled trial; CCT $=$ controlled clinical trial]

\section{DECLARATIONSOF INTEREST}

The authors declare that they have no conflict of interest. 